\title{
Keyword index to volume 35
}

AAC see Acute acalculous cholecystitis Abstracts for Spinal Cord 865

$\alpha$-Actin 26

Activities of daily living 850

Acute acalculous cholecystitis 124

Adjustment, social 320, 349

ADL see Activities of daily living

Admissions, early, delayed and late 147

Ageing 818

Ambulation 292, 580, 585

American Spinal Injury Association 358, 368

Amputation, bilateral below-knee 121

Anaemia 58

Anaesthetics 647

Angiography, spinal 624

Ankylosing spondylitis 550

Anterior column 286

Anxiety 516

AO Thoracolumbar Locking Plate 286

Arachnoiditis 629

ASIA see American Spinal Injury Association

Assessment 171

functional 747,850

quality-of-life 17

ATLP see AO Thoracolumbar Locking Plate

Audit 147

Axon growth 469

Axotomy 474

Bacteriuria 605

Birth, healthy live 179

Bladder autoaugmentation 456

Bladder function, urinary 368

Bladder outlet obstruction 48

Blood pressure 647

BMD see Bone mineral density

BMP (bone morphogenetic protein) 740

Bone mass loss 223

SCI associated 374

Bone mineral density 223,374

Bone morphogenetic protein (BMP) 740

Bone scintigraphy 158

Bony fusion 777

BOO see Bladder outlet obstruction

Book reviews 129, 258-9, 785-6

Bowel programme 394

Breathlessness 652

Brown-Sequard syndrome 554

Calcium 521

Calorimetry, indirect 599

Cardiac arrest 181

Cardiac rehabilitation 181

CAT see Choline acetyltransferase

Catecholamine 647
Cathartics 394

Catheterisation

intermittent self- 613

urethral 229

urinary 521

Catheters 229

Cauda equina compression 704

Causes of spinal cord injury 809

Central pontine myelinolysis 332

Cerebral lesion 487

Cervical spinal cord 729

Cervical spinal cord injury 181

incomplete 752

Cervical spine 700,857

volume incrementation 674

Cervical spondylosis 768

Choline acetyltransferase 729

Chronic inflammatory state 58

Classification, pain 69

Classification of SCI, International Standards 266, 275

Clinical neurological correlation 213

Colonization, urethral 282

Compensation 349

Compliance 314

detrusor 456

Complications 608

digestive 124

medical 147

urological 308

Compression

cauda equina 704

chronic 729,740

Computed tomography 92

three-dimensional 674

Constipation 53, 394

chronic 116

Consulting 415

Corrigenda 862-4

Corticospinal tract 205

Cough 299, 540, 652

CPM see Central pontine myelinolysis

Creatinekinase 234

Croatia 620

CT see Computed tomography

Customer satisfaction 109

Cysts, cervical neurenteric 700

D. dimers 151

Death see Mortality

Decompression, anterior 777

Denervation, partial 752

Depression 516

Detrusor compliance 456

DEXA see Dual energy X-ray absorptometry

Diet 116

Dilatation, balloon 96
Dimers, D. 151

Disability 850

Dual energy X-ray absorptometry 223 , 374

Dysaesthesia 446

Dysreflexia, autonomic 390, 647

Dyssynergia, detrusor-sphincter 96

E. coli see Escherichia coli

Economics of spinal cord injury 809

Editorials 135, 264-5, 409-10, 717, 793-5

Ejaculation 463

Elbow joints 308

Electrical stimulation 1, 540, 546

Electroejaculation 179, 463

Electromyography $171,308,768$

Electrophysiology 176

Embolisation 624

EMG see Electromyography

Employment 238

$\beta$-Endorphin 757

Endothelial cells 26

Energy costs 121

Enterococcus spp. 282

Epiconus syndrome 163

Epidemiology 76, 253, 361, 420, 509, 53] 809

EPM see Extra pontine myelinolysis

Equipment, orthopaedic 139

Erectile dysfunction 99

Escherichia coli 282

Etidronate 158

Evoked potentials, somatosensory 368

Exercise 1

cardiovascular 680

electrical stimulation-induced 680

Exercise cycle 176

Exercise testing 503

Extra pontine myelinolysis 332

F-waves 176

Fertility 463

FES see Functional electrical stimulation

Fibrillation, ventricular 256

Fibrinolytic reactivity 151

FIM see Functional independence measure

Fixation, internal spinal 286, 429

FNS see Functional neuromuscular stimulation

Foramen magnum 554

Foraminotomy 725

Function 245

orthosis aided 217

Functional assessment 747, 850

Functional electrical stimulation 569,585 647 
Functional independence measure 22, 386 Functional neuromuscular stimulation 286

\section{Gait 580}

Ganglion cyst 632

Gabapentin 171, 686

Germany 411,415

Gunshot wounds 341

Haemangioma, vertebral 624

Hand grasp 569

Health 818

Health expectancy 818

Health services research 109

Health status 109, 326

Heart rate 647

Herniation

double-level disc 725

extraforaminal invertebral disc 725

lumbar disc 757

multiple disc 183

Hips 443

Histochemistry 86

Hodgkin's disease, spinal epidural involvement 704

Home care for severely disabled 40

Hormones, antidiuretic 303

Hyperextension injury 857

Hyperreflexia, autonomic 256

Hypertonicity, external urethral sphincter 690

Hyponatraemia 303, 332

Hypotension, postural 303

Impairment scale and protocol, ASIA 358, 368

IMSOP see under International Medical Society of Paraplegia

Incidence of spinal cord injury 253, 531

Incontinence, urinary 546

Independent living 326

Inflammatory state, chronic 58

Intensive care, neuro- 565

International Medical Society of Paraplegia 796-7

AGM 130-1

medallists 1996 and 1997 187-8, 859

President 335

International Standards Classification of SCI 266, 275

Investigation, microbiological 534

IRGO see Orthoses

Isolation 617

Japan 718,720

Jordan 253

\section{K complex 248}

Labour Welfare Corporation (Japan) 718

Lactatedehydrogenase 234

Laminectomy, decompressive 624

Laminoplasty 674

Leg movements, periodic 248

Length of stay 147

Letters to the Editor 64, 189-91, 402-3,
$557-8,708-11,860-1$

Ligamentum flavum 740

Litigation 349

Living conditions 487

Lumbar canal stenosis 757

Lumbar cerebrospinal fluid pulse wave 735

Lumbar disc herniation $183,725,757$

Lumbar radiculopathy 725

Lumbar spine 632

Lung function 503

Magnetic resonance imaging 361,664 , 704,773

Magnetic transcranial stimulation 205

Management

initial and subsequent 565

pain 658

of pressure sores 641

respiratory 805

Medicine, history of 139

Meetings and Notices 65-7, 132-4, 194-6, 260-2, 336-8, 404-6, 482-4, $559-61,636-8,712-14,787-9$, 866-8

Meningitis, tuberculous 629

Meta-analysis 829

Metabolism 599, 680

Methicillin-resistant Staphylococcus aureus, psychological impact 617

Methylprednisolone, ultra-high administration 420

Microsurgery 725

Mobility 574

Mortality 76, 818

Motor cortical mapping 205

Motor neuron lesions, upper and lower 37

Motor score 386

Motorneurons 752

Mouse, twy (tiptoe-walking Yoshimura) 729

Mouth pressure, static 99

MRI see Magnetic resonance imaging

MRSA see Methicillin-resistant Staphylococcus aureus

Multicentre study 446

Multiple sclerosis 33,613

Muscle damage 234

Muscle fibre type grouping 752

Muscle flaps 437

Muscles 308, 546

skeletal 1,86

vastus lateralis 437

Myelin 469

Myelography 361

Myelopathy

acute onset 773

cervical 757,763

chronic compression 740

postural hypotension 303

thoracic 777

Myoclonus 248

Myoglobin 234

Myosin isoforms 86

National Rehabilitation Centre for the
Disabled (Japan) 720

Neoplasms 554

resection 624

spinal 429

Neurogenic voiding dysfunction 456

Neuroimaging 163

Neurological deficit 92

Neurological disorders 139

Neurological examinations 275

Neurological recovery 386,420

Neurological State and Function, International Standards for 358

Neurontin 171

Neuropathic bladder $33,48,213,613$

Neuropathic bowel, faecal impaction 694

Neuropathic rectum 53

Neuroprostheses, eligibility criteria 569

Nitrogen balance 599

Nitroglycerin, transdermal 99

Nutrition 599

Obesity 245

Obituaries 193

Occlusion, venous 151

Operations, results 443

Operations, traumatological 420

OPLL see Ossification of posterior longitudinal ligament

OPS see Organic psychic syndrome

Organic psychic syndrome 487

Orthoses 217

gait 585

isocentric reciprocal gait (IRGO) 580

reciprocating gait 121,383

walkabout 574,580

Orthostasis 303

Osmolality of catheters 229

Ossification, heterotopic 158, 443

Ossification of posterior longitudinal ligament 550

extrapleural approach 777

Osteochondroma, huge solitary 773

Outcomes

bowel management 608

of orthoses 574

surgical 763

Oxygen therapy, hyperbaric 763

Oxygen uptake 121

Paediatric spinal cord injury 747

Pain

acute and chronic 446, 658,814

classification 69

myofascial syndrome 658

neuropathic 658

objective evaluation 757

prevalence 814

psychology of 814

sites 814

surgery and 526

Papaverine, intracavernous 99

Paraparesis 550

Paraplegia 1, 22, 76, 116, 121, 176, 223, $248,286,299,390,443,446,469$, $487,493,620,641,652,768$

Paraplegic patients $383,437,580,585$ 
Pathology 857

Peak expiratory flow rate 299

Pericyte 26

pH 521

Phenol 690

Phrenic nerve stimulation/mechanical ventilation 40

Physical activity 248

PIE see Pulsed irrigation evacuation

Poliomyelitis 503

Post-poliomyelitis syndrome 503

Posterior longitudinal ligament 632

Pressure sores 58

management 641

prevalence 595

prevention 590,595

sacral 437

Prevention of spinal cord injuries 253, 509

Prognosis factors 664

Psychogenic disorder 700

Psychological distress 516

Psychology of pain 814

Psychometric analysis 17

Pudendal nerve block 690

Pulmonary infection 43

Pulsed irrigation evacuation 694

Quality of life $326,456,829,841$

assessment 17

health related 747

Questionnaire, short-form 17

Radiculopathy $632,725,757$

Rate of perceived exertion 292

Readmissions, causes of 415

Rectal inertia 53

Rectal reinnervation 53

Red nucleus 474

Regeneration, neurotrophic factors 469

Rehabilitation 620

hospital 565

orthotic 217

postpolio 503

and table tennis 61

using treadmill 383

vocational 238, 493

Reintegration, vocational 493

Research 565

health services 109

Respiratory capabilities 540

Respiratory insufficiency 40

Respiratory management 805

Respiratory muscle fatigue 99

Respiratory muscle strength/weakness 43 , 99

Respiratory muscle training 540

Respiratory pressures, static 43

Respiratory symptoms 652

RGO see Orthoses

RPE see Rate of perceived exertion

Schwannoma 554

SCIM see Spinal Cord Independence Measure
Scintigraphy, bone 158

Self-care 308

Semen retrieval 463

Sensory score 386

Serum protein alteration 58

Service delivery 109

Sexuality 136

Skeletal muscles 1, 86

Sleep 248

Social support 487

Somatosensory evoked potentials 368

Spasticity 171, 686

Special centres, capacity of 415

Sperm collection devices 463

Sperm injection, intracytoplasmic 179

Sphincterotomy 96

external 48

Spinal canal, volume incrementation 674

Spinal canal narrowing 92

Spinal cord 26, 341, 740

termination of 163

Spinal cord blood flow 735

Spinal cord compression 183, 624, 740

Spinal cord diseases 361

Spinal Cord Independence Measure, validity and reliability 850

Spinal cord injured patients 605

Spinal cord injuries $1,17,22,37,40,43$, $69,76,86,96,99,109,116,124$, $136,147,151,158,171,179,198$, $205,213,217,223,229,238,253$, $256,275,282,292,299,303,314$, $320,326,341,349,358,368,374$, $386,390,394,415,446,463,474$, $487,509,516,526,531,534,546$, $574,590,595,599,608,617,620$, $641,647,652,658,664,686,690$, $694,720,747,809,814,818,829$, 841

Spinal cord injury unit 565

Spinal cord lesions $48,99,411$

Spinal fraction 139

Spinal Injuries Centre 718

Spinal Service profile 720

Spine fracture 664

Spondylosis, cervical 768

SSEP see Somatosensory evoked potentials

Stabilisation of spine posterior vertebral 429 surgical 420

Standing 574, 585

Stenosis, lumbar canal 757

Substance P 757

Surgery

anterolateral approach 183

outcomes 763

and pain 526

for pressure sores 641

spinal 429

stabilization of spine 420

Survival curves 76

Swimming pools 509

Symptomatology, neurological 163
Syringomyelia 629

Taiwan 841

Teaching 565

Tetraplegia 1, 22, 40, 43, 61, 99, 217, 223 $245,299,308,332,358,390,394$. $446,487,569,620,652,805$

Tetraplegic patients 540

Therapy see Treatment

Thoracic spine 550, 773, 777

Thoracolumbar spine 163, 286

Threshold loading, incremental 99

Thrombosis 151

Tissue transplantation 546

Transplantation, tissue 546

Treadmill 383

Treatment

comprehensive 411

hyperbaric oxygen 763

organisation 411

physical 176

present situation 411

pressure sores 641

prophylactic antimicrobial 605

special 415

surgical 526

trends of origin 415

Trochanteric region 437

Turkey, south-east 531

Twy (tiptoe-walking Yoshimura) mouse 729

Ultrasound 613

Unemployment 238

Urethral colonization 282

Urinary tract abnormalities 33

Urinary tract infection

antimicrobial therapy for 605

polymicrobial 534

prevention 198

urethral cultures 282

Urine 521

Urodynamics $37,213,546$

Urology 498

UTI see Urinary tract infection

Vascular corrosion cast 26

Vascular disorders 437

Vasoactive substances 390

Vasomotion 26

Vastus lateralis muscle 437

Vertebral canal bony fragments 92

Vertebral fractures 92

Vibrostimulation 463

Violence 341

Vital capacity 805

$\mathrm{VO}_{2}$ see Oxygen uptake

Voiding dysfunction, neurogenic 37

Wheelchair cushions 590

Wheelchair propulsion 234

Wheelchair use 768

Women 136

urethral cultures in 282 